\title{
Meshed multipath routing with selective forwarding: an efficient strategy in wireless sensor networks
}

\author{
Swades De ${ }^{\mathrm{a}, *}$, Chunming Qiao ${ }^{\mathrm{b}}$, Hongyi $\mathrm{Wu}^{\mathrm{c}}$ \\ ${ }^{a}$ Department of Electrical Engineering, State University of New York at Buffalo, Buffalo, NY 14260, USA \\ ${ }^{\mathrm{b}}$ Department of Computer Science and Engineering, State University of New York at Buffalo, Buffalo, NY 14260, USA \\ ${ }^{\mathrm{c}}$ The Center for Advanced Computer Studies, University of Louisiana at Lafayette, Lafayette, LA 70504, USA
}

\begin{abstract}
Due to limited functionalities and potentially large number of sensors, existing routing strategies proposed for mobile ad hoc networks are not directly applicable to wireless sensor networks. In this paper, we present a meshed multipath routing (M-MPR) protocol with selective forwarding (SF) of packets and end-to-end forward error correction (FEC) coding. We also describe a meshed multipath searching scheme suitable for sensor networks, which has a reduced signaling overhead and nodal database. Our performance evaluations show that (1) M-MPR achieves a much improved throughput over conventional disjoint multipath routing with comparable power consumption and receiver complexity; (2) to successfully route a message using FEC coding, selective forwarding (SF) consumes much less network resources, such as channel bandwidth and battery power, than packet replication (or limited flooding).
\end{abstract}

(C) 2003 Elsevier B.V. All rights reserved.

Keywords: Sensor network; Meshed multipath; Selective forwarding; Forward error correction coding; Energy efficiency; Performance analysis

\section{Introduction}

Miniaturization of processing and memory devices and their affordable cost have opened up a new paradigm of remote information access and

\footnotetext{
This paper was presented in part at WCNC 2003, New Orleans, LA, March 2003 and at ICC 2003, Anchorage, AK, May 2003.

${ }^{*}$ Corresponding author. Tel.: +1-716-645-5084x15; fax: +1716-645-3464.

E-mail addresses: swadesd@cse.buffalo.edu (S. De), qiao@cse.buffalo.edu (C. Qiao), wu@cacs.louisiana.edu (H. $\mathrm{Wu})$.
}

control using sensor networks [2,7,9]. A wireless sensor network is similar to mobile ad hoc networks, but it differs from them in that the sensors have much reduced capabilities, such as limited transmission range, limited or no mobility, and limited battery power [1]. In addition, in many applications, such as remote field status monitoring, the field sensors may be located close to ground, thus causing ground wave absorption. Also, multiuser interference caused by densely populated sensors may lead to a high packet error rate. Therefore, existing MANET routing approaches (e.g., [8,10,14,21,25,27]) may not work well, and new techniques need to be developed. 
While retransmissions can be used to recover from data loss, basic sensors may not have enough storage space to save the collected information for necessary retransmission. Moreover, hopby-hop retransmission based on either promiscuous listening to the neighbor's transmission [14], or acknowledgment (or negative acknowledgment) from downstream neighbors [12,29] requires additional receive power and introduces delay in trans-to-receive mode changeover. To facilitate fast and successful end-to-end delivery of information, we propose to set up meshed multiple paths from a source (e.g., a field sensor) to a destination (e.g., a data collection/processing center).

Among the possible variants, there are two ways of effecting disjoint multipath routing (MPR) in multihop networks: (1) Each packet is sent along different disjoint routes (see e.g., [3,4,19, $22,28])$. The decision on which path to use is made by the source on a packet-by-packet basis. We will call such an approach disjoint (or split) MPR (DMPR) with selective forwarding (SF). (2) Multiple copies of a data packet are transmitted simultaneously along multiple disjoint routes from a source to a destination (see, e.g., [12,17]). Such an approach will be called D-MPR with packet replication (PR) (or limited flooding). In Section 5, other related approaches including what we call preferential routing, where one or more secondary routes that are either disjoint or non-disjoint (also termed meshed/braided) with the primary route are kept stand-by to recover from any failure of the primary route (see, e.g., $[8,12,14,23])$, will be described.

A forward error correction (FEC) coding scheme can be adopted in all of the above routing approaches. When FEC is employed, the second approach (D-MPR with PR) would require the minimum code length (and hence the least error correction overhead), but it may be inefficient with regard to resource utilization (as more transreceive power is wasted and less traffic is served). The first approach (D-MPR with SF) completely relies on the end node (e.g., the source) to make a routing decision for every packet. Due to network dynamics (such as time-varying number of active nodes and their locations), the route information available at an end node may not be up-to-date. Moreover, in wireless sensor networks, it is not feasible to exchange the entire network information among all nodes. Therefore, the routing decision taken at an end node will not be well-informed and in fact is prone to be ineffective.

In this paper, we aim at reliable and efficient routing in sensor networks. We present a meshed multipath routing (M-MPR) scheme, which allows some (if not all) intermediate nodes to have more than one forwarding direction to a given destination. In addition, we propose selective forwarding of packets (SF) where the forwarding decision is taken dynamically, hop-by-hop, based on the conditions of downstream forwarding nodes. Endto-end FEC coding is also used to avoid acknowledgment-based retransmission. A new mesh-based multipath searching scheme, which requires a lower control overhead and a smaller nodal database than tree-based (e.g., in [8,27]) and sequential (e.g., in [12]) searching approaches, is also described. For completeness, we will touch upon issues related to mesh-based route discovery and routing protocols, but our main focus in this paper will be on the performance evaluation of the proposed M-MPR with the SF strategy, and its comparison with other approaches such as DMPR-SF, D-MPR-PR, and M-MPR-PR.

Based on our evaluation, we draw the following conclusions: (i) In terms of throughput, M-MPRSF outperforms D-MPR-SF. (ii) Throughput gain of M-MPR-SF is greater for longer end-to-end distance. (iii) To successfully route a message to the destination, PR has substantially higher resource requirements than SF, along either disjoint or meshed multipaths.

The rest of the paper is organized as follows. In Section 2, our proposed M-MPR with SF scheme is introduced and the associated mesh-based multipath searching approach is described. Section 3 contains throughput analyses of M-MPR and DMPR with PR and SF, respectively. Numerical and simulation based performance results in terms of throughput gain, receiver complexity, and battery power usage are presented in Section 4 . Related work is surveyed in Section 5, and finally, Section 6 concludes the paper. 


\section{Meshed multipath routing}

In this section, the steps for meshed multipath formation are outlined. Two possible variants of packet forwarding schemes (PR and SF) are also described.

\subsection{Multipath searching}

In sensor network applications, such as remote field status monitoring, the field nodes primarily need to communicate with a common monitoring and control center, which could also be a clusterhead (henceforth called the controller node). We envisage that in such applications, the field sensors would be mostly stationary, and their location information can be imparted during the initial deployment phase via standard trilateration approach using other GPS-capable nodes [11] or via the directional beaconing approach described in [24]. The controller node, which may be capable of limited movement but is mostly stationary, is also location aware and can make its location information known to the field sensors (e.g., via broadcast or beaconing) whenever it relocates itself. With the above considerations, a meshed multipath is set up in the following three steps.

Acquiring neighborhood information: Once deployed and localized, each active node broadcasts its ID, residual battery power, and location information to local neighbors. Thereby, each active node gathers the local neighborhood information. For each active neighbor $i$, a node maintains the following information in its database: $\left\{I D_{i}\right.$, location $_{i}$, residual_power $\}$. Note that since the field nodes are assumed stationary, no periodic update of neighborhood status is necessary. In other words, unless there is any change in local neighbors' status, e.g., a node is going into sleep mode or has just woken up, the local neighborhood database does not need an update. Any such change of a node's status is locally broadcast, based on which of the neighborhood tables of nearby nodes are updated.

Route discovery: Based on the current neighborhood database and location information of the controller node, each of the field nodes tries to form a meshed multipath to it. To this end, an

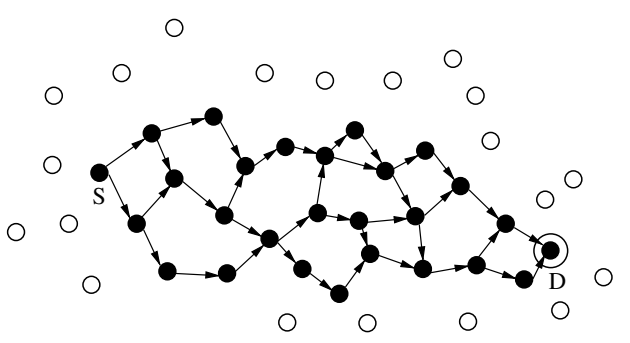

(a)

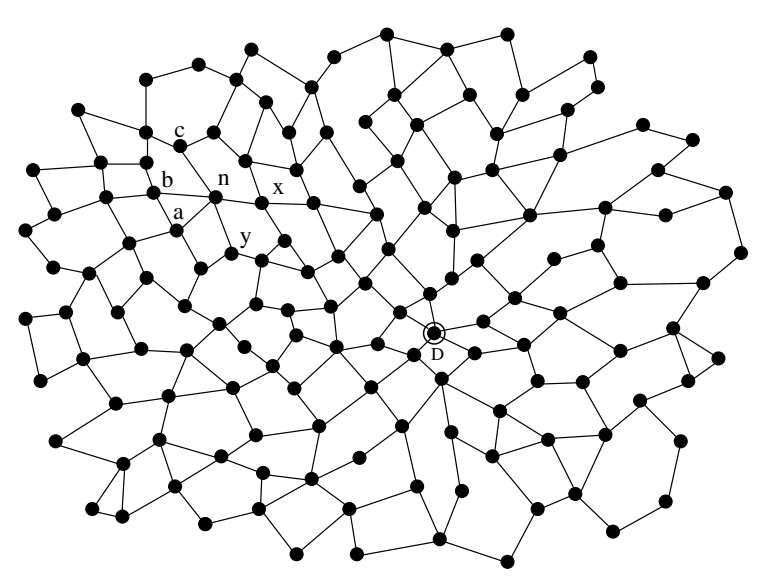

(b)

Fig. 1. Pictorial views of meshed multipath: (a) a source-todestination meshed multipath and (b) meshed topology formed by many-sources-to-a-destination routes.

intermediate node is allowed to accept (and record) more than one discovery packet. Typically, to reduce the receiver complexity ${ }^{1}$ and power consumption of a node, for a source-to-destination route discovery process, at most two copies of a discovery packet are accepted by an intermediate node and one (the first arrival) is forwarded to maximum two downstream neighbors (see Fig. 1(a)). We choose maximum two forwarding nodes as in [10], where it was observed that a maximum of two forwarding links at a node allow just enough flexibility for selecting an alternate route with a minimum possible additional control overhead.

\footnotetext{
${ }^{1}$ Receiver complexity of a node is a function of the number of incoming links.
} 
A route discovery packet has the following fields: \{source_ID, source_location, intermediate_ node_ID, next_node_ID, next_node_ID , destination_ID, destination_location, TTL $\}$. The IDs of forwarding nodes (next_node_ID $, i=1,2$ ), intermediate_node_ID, and $T T L$ values are updated at each intermediate stage. The TTL (time-to-live) value is slightly greater than an estimated hop count to the destination, which is set such that if a discovery packet fails to reach the destination it is dropped after the TTL expires. Each intermediate node maintains the following information in its routing database: \{previous_node_ID $1, \ldots$, previous_node_ID ${ }_{n}$, next_node_ID, next_node_ID $\}$. Note that since there are many peripheral field nodes trying to reach the same destination (the controller node), an intermediate node can have more than two "previous_node" entries in its routing table, although there will be no more than two "next_nodes" (see Fig. 1(b)). However, the list of "previous_node" does not grow indefinitely, as (i) the number of local neighbors is finite and (ii) no new entry in the routing table is made for a discovery packet coming from an upstream neighbor which is already listed in the list. If an intermediate node, which has already forwarded a discovery packet to the destination, receives another discovery packet, it just updates the previous_node list (for sending back the route reply packet) in its routing table and drops the packet. It may be noted that in some cases, due to the nodes' random placement and/or due to its neighbors' states, it is not necessary that all the nodes have two forwarding neighbors all the time, although a node is (or a group of nodes are) assumed to be connected to the rest of the network.

An entry in the routing table at a node is maintained as a soft-state, which is deleted after a time out unless it receives a reply from the controller node. Since sensor applications are mostly data-centric, jitter (delay differences) between packet arrivals is not a major concern. Therefore, apart from storing and maintaining upstream and downstream nodes' information, no other resource reservation is made during the route discovery phase. Hence, the discovery process can also be considered as a topology construction process.
Route reply: This message is necessary to notify which of the nodes, involved in route discovery, actually constitute the meshed multipath. Corresponding entries at all other nodes involved in the previous Route discovery process will eventually disappear (upon expiration of the soft-state). When the controller node receives the discovery packets from a single source, it selects the first two of them and sends a route reply following the original links used by Route discovery packets (but in reverse direction) with the following fields: \{source_ID, source_location, intermediate_node_ $I D$, previous_node_ID 1 , previous_node_ID 2$\}$. Each intermediate node changes the state of its corresponding entries from 'soft" to permanent (as long as the node remains active and connected), updates the fields of the reply packet other than the source information, and forwards the reply packet to its upstream node (towards the source). Note that in forwarding the route reply message, a node does not need to know the source information. If the discovery packets from many sensor nodes arrive via a common path to the controller node, the sensor nodes are replied back via a multicastbased reply.

After the meshed network topology is formed, a node along the meshed multipath has the responsibility to remain connected. If an intermediate node goes out of service (due to battery drainage), or goes to sleep mode as a power saving measure, the upstream nodes select appropriate neighbors (and if needed, discover routes) to remain connected. However, intermittent "link breakage" due to, e.g., interference is not considered a form of disconnection and will not trigger reconfiguration of the meshed multipath. Rather, it will be handled using selective forwarding (SF) as will be described later.

From a sensor node's view point, a typical meshed multipath to the destination is as shown in Fig. 1(a). From a group of nodes' view point, the meshed multipaths to the controller node $(D)$ is as shown in Fig. 1(b). Observe that in the constructed meshed topology the number of downstream links is no more than $t w o$, but the number of upstream nodes can be more. For example, in Fig. 1(b), the node $n$ has three upstream nodes: $a, b$, and $c$; and two downstream nodes: $x$ and $y$. 


\subsection{Multipath routing}

After the meshed multipath is constructed, the data packets can be forwarded (using the routing table built in route searching phase at each active node) to the destination along the meshed multipath using either packet replication (PR) or selective forwarding (SF).

In PR, a packet from a source is copied along all possible paths to its destination. To reduce power consumption due to transmission of multiple copies of the same packet, a node receiving more than one correct copy of the packet from upstream nodes filters out one successful packet for forwarding to the downstream nodes.

On the other hand, in SF, if more than one downstream nodes are available at either the source or an intermediate node, the packet is forwarded along only one downstream link based on local conditions (e.g., health of the downstream nodes). If all outgoing links from a node are equally good, one is selected randomly. Besides achieving fault tolerance, such selective forwarding along the meshed multipath is more efficient than PR in terms of resource utilization and congestion avoidance. It can also distribute the traffic among multiple routes and conserve the energy among different nodes more evenly than preferential routing $[8,12,14,23]$. Also, this packet distribution policy automatically refreshes a node's association with the mesh, thereby minimizing the need for explicit route maintenance.

It may be noted that while the signal transmitted by a simple sensor node is generally broadcast to all its neighbors, the major difference between PR and SF is that in the former, the packet is intended for multiple neighbors, each of which will receive and forward the packet whereas in the latter, only one receiver will receive and forward. On the other hand, because of the broadcast nature, meshed multipath routing (M-MPR) does not require any extra transmission energy when compared to disjoint multiple path routing (DMPR) and hence is a natural choice. Moreover, M-MPR introduces more flexibility than D-MPR in making selective forwarding decision, thereby increasing the chance of successful packet delivery. Nevertheless, to minimize possible medium access conflict, M-MPR would require either a tunable receiver (implying more delay in channel access) or more fix-tuned receivers (implying additional orthogonal codes). In the rest of the paper, we will not consider any further details of routing and MAC protocol aspects. Rather, our focus will be the performance evaluation of our proposed approach and its comparison with other similar approaches.

\section{Throughput analysis}

We now evaluate the throughput performance of M-MPR and D-MPR schemes with PR and SF, respectively. In our analysis we have also considered tree-based multipath routing, as proposed in the literature (see, e.g., [20]). Its throughput is in between D-MPR and M-MPR performance, the intuition being that, unlike in M-MPR, its routing flexibility from a source is not extended all the way to the destination. In this paper, we will restrict our scope to D-MPR and M-MPR.

In analyzing the throughput for a source-destination pair, we do not consider FEC coding, and if FEC coding is used, we do not distinguish the data packets (blocks) from possible error correcting blocks. We define Normalized throughput $(T)$ as the probability of successful arrival of a packet to the destination. The source-to-destination hop length is denoted by $H$, where all routes are assumed to be of equal length and the meshed multipath is mostly regular (see Figs. 2 and 3). Note that although the "equal length routes" and "regular mesh" assumptions may not be very practical, with these assumptions, the system lends itself to tractable analytic performance evaluation which can be used to gain intuitive understanding

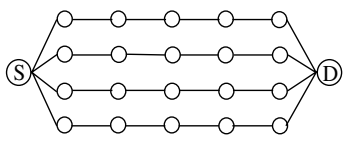

(a)

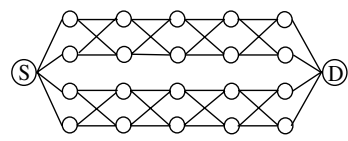

(b)
Fig. 2. Examples of 6-hop multiple routes: (a) disjoint multipath and (b) its node-equivalent meshed multipath (to be discussed later in Section 4.2.2). 


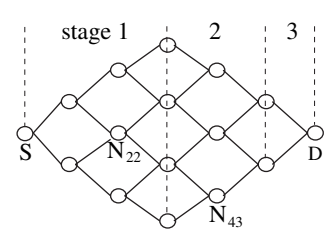

(a)

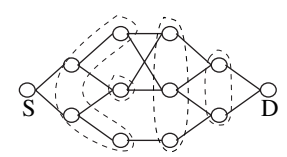

(b)

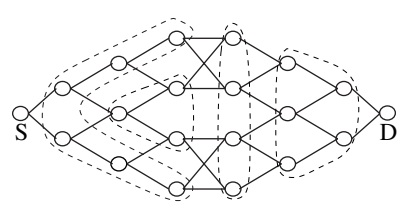

(c)

Fig. 3. Examples of meshed multipath: (a) even number of hops ( $H=6$ ); (b) odd number of hops $(H=5),\lfloor H / 2\rfloor$ is even and (c) odd number of hops $(H=7),\lfloor H / 2\rfloor$ is odd.

of routing performance. In Section 4, we will study the performance under more practical assumptions via simulations, where due to random location of field sensors, not all routes between a source to the destination are of equal length. In addition, for MMPR, not all intermediate nodes will have two incoming as well as two outgoing links.

With the above simplified assumptions, the number of nodes associated with $r$ disjoint $H$-hop source-destination routes in D-MPR, including source and destination, is

$N^{(d)}=r(H-1)+2$.

On the other hand, with maximum two incoming or outgoing branches at each node (see Fig. 3), the number of nodes involved in M-MPR is

$N^{(m)}= \begin{cases}((H+2) / 2)^{2}, & H \text { even, } \\ \lceil H / 2\rceil(\lceil H / 2\rceil+1), & H \text { odd }\end{cases}$

Hereafter, for each packet transmission, link error and intermediate node failure probabilities are denoted by $p_{l}$ and $p_{n}$, respectively. While $p_{l}$ captures Gaussian channel noise as well as the error due to medium access conflict, $p_{n}$ captures the packet loss due to input buffer overflow and node failure. Note that, to highlight the differences between different multiple path routing schemes, the end node (i.e., the destination) is considered ready to receive (i.e., $p_{n}=0$ ) all packets.

In our analysis, a link is modelled as an additive white Gaussian noise (AWGN) channel. If $p_{b}$ is the average bit error probability (or BER) due to channel error and $B$ is the packet size (number of bits), then

$p_{l}=1-\left(1-p_{b}\right)^{B}$.

For direct sequence spread spectrum (DS-SS) based channel access, with $K$ contending nodes and $C$ chips per bit, Gaussian approximation [26, p. 282] yields the average BER (using conventional matched filter receiver):

$p_{b}=Q\left(\frac{1}{\sqrt{\frac{K-1}{3 C}+\frac{N_{0}}{2 E_{b}}}}\right)$,

where $E_{b} / N_{0}$ is the signal-to-noise ratio per bit.

\subsection{Packet replication $(P R)$}

We now consider the normalized throughput performance with the PR approach.

\subsubsection{Disjoint multipath ( $D-M P R-P R)$}

Fig. 2(a) shows an example of a set of 4 disjoint routes, each of which is 6 hops long. In D-MPRPR with $r$ parallel $H$-hop routes, the normalized throughput $T_{\mathrm{PR}}^{(d)}$ can be obtained as

$T_{\mathrm{PR}}^{(d)}=1-\left[1-\left(1-p_{l}\right)^{H}\left(1-p_{n}\right)^{H-1}\right]^{r}$,

where $\left(1-p_{l}\right)^{H}\left(1-p_{n}\right)^{H-1}$ is the probability of successful delivery of a packet along a particular route.

\subsubsection{Meshed multipath ( $M-M P R-P R)$}

There could be different ways of forming meshed multipaths. To facilitate a fair comparative analysis, we first consider three examples of meshed multipath as shown in Fig. 3. How the stages are divided will be discussed later.

We denote the intermediate nodes by $N_{i j}$ where $i$ stands for the hop length from source and $j$ stands 
for its position from the top of the mesh (see nodes $N_{22}$ and $N_{43}$ in Fig. 3(a) for example). Correspondingly, successful packet arrival probability at the $(i, j)$ th node is denoted by $P_{i j}$. Depending on the hop length, there are three possible cases of meshed multiple routes: (a) even $H$, (b) odd $H$, even $\lfloor H / 2\rfloor$, and (c) odd $H$, odd $\lfloor H / 2\rfloor$. Referring to Fig. 3, there can be up to four categories of intermediate nodes: (i) The nodes having only one predecessor node. For example, in Fig. 3(a), these are the nodes $N_{i j}$, where $(i, j)=(1,1),(1,2)$, $(2,1),(2,3),(3,1),(3,4)$. In general, $i=1,2, \ldots$, $\lceil H / 2\rceil$ and $j=1$ or $j=i+1$. The nodes belonging to different categories are marked in Fig. 3(b) and (c). (ii) The remaining nodes in the left half of the mesh (i.e., the nodes with $1<i \leqslant\lfloor H / 2\rfloor$ and $1<j<i+1)$, which have two predecessor nodes. In Fig. 3(a), the nodes $N_{22}, N_{32}$ and $N_{33}$ belong to this category. (iii) For odd $H$, the nodes $N_{\left[\frac{H}{2}\right], j}$, where $1 \leqslant j \leqslant\lceil H / 2\rceil$. Note that there is no category (iii) node in Fig. 3(a) (where $H$ is even). (iv) All other nodes in the right half of the mesh except the destination, i.e., the nodes from $\lceil H / 2\rceil+1$ hop to $H-1$ hop. In Fig. 3(a), the nodes 4-hop and 5-hop away from the source fall in this category.

For category (i) nodes: A packet will successfully reach node $N_{i j}$ if $N_{i j}$ is ready to receive, and its incoming link is error-free during transmission of the packet. That is,

$P_{i j}=\left(1-p_{l}\right)^{i}\left(1-p_{n}\right)^{i}$.

Note that $P_{i j}$ is a function of $i$ only, i.e., the hop distance of $N_{i j}$ from $S$.

For category (ii) nodes: $P_{i j}$ is recursively obtained as:

$$
\begin{aligned}
P_{i j}=\left(1-p_{n}\right) & {\left[1-\left(1-\left(1-p_{l}\right) P_{i-1, j-1}\right)\right.} \\
& \left.\times\left(1-\left(1-p_{l}\right) P_{i-1, j}\right)\right] .
\end{aligned}
$$

Here, $\left(1-p_{n}\right)$ is the probability that the node $N_{i j}$ is ready to receive. The remaining term within the parenthesis is the successful packet arrival probability from at least one incoming directions, given that $N_{i j}$ is ready to receive.

For category (iii) nodes ( $H$ odd): In this category, depending on whether $\lfloor H / 2\rfloor$ is even (as in Fig. 3(b)) or odd (as in Fig. 3(c)), $P_{i j}$ is recursively obtained as shown below.

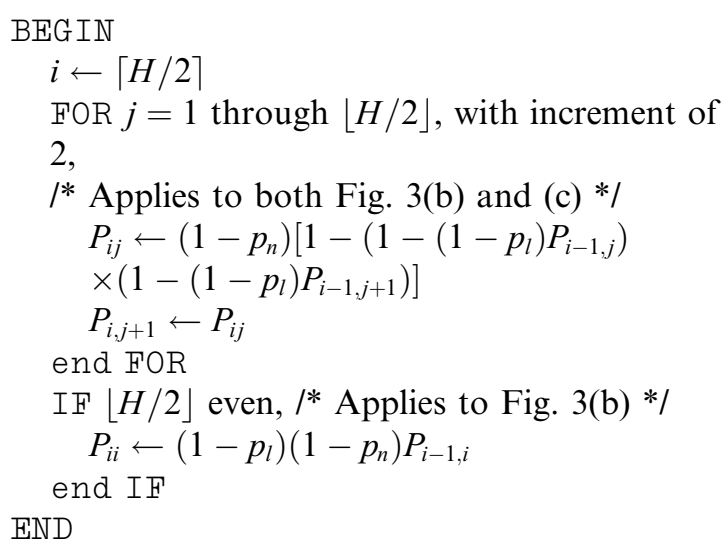

For category (iv) nodes: All nodes in this category (like the category (ii)) have two predecessor nodes node. The corresponding $P_{i j}$ is given by

$$
\begin{aligned}
P_{i j}=\left(1-p_{n}\right)[ & 1-\left(1-\left(1-p_{l}\right) P_{i-1, j}\right) \\
& \left.\times\left(1-\left(1-p_{l}\right) P_{i-1, j+1}\right)\right] .
\end{aligned}
$$

By determining the $P_{i . j}$ 's for nodes in categories (i)-(iv), we obtain the probabilities $P_{H-1,1}$ and $P_{H-1,2}$. Finally, the end-to-end successful arrival of a packet, or normalized throughput in M-MPR$\mathrm{PR}$ is given by

$T_{\mathrm{PR}}^{(m)}=1-\left(1-\left(1-p_{l}\right) P_{H-1,1}\right)\left(1-\left(1-p_{l}\right) P_{H-1,2}\right)$.

Note that the above is similar to $P_{i j}$ for the nodes in categories (ii) and (iv), except that the destination node is presumed ready to receive all packets.

\subsection{Selective forwarding ( SF)}

Below, we analyze normalized throughput with selective forwarding (SF) of packets.

\subsubsection{Disjoint multipath ( $D-M P R-S F)$}

In D-MPR-SF, route selection can be done only at the source. The corresponding normalized throughput is thus given by

$T_{\mathrm{SF}}^{(d)}=\left(1-p_{l}\right)^{H}\left(1-p_{n}^{r}\right)\left(1-p_{n}\right)^{H-2}$,

where $\left(1-p_{l}\right)\left(1-p_{n}^{r}\right)$ is the probability of reaching to a next node from the source, and $\left(1-p_{l}\right)^{H-1}\left(1-p_{n}\right)^{H-2}$ is the probability of successfully covering the remaining $(H-1)$ hops. 


\subsubsection{Meshed multipath ( $M-M P R-S F)$}

Referring to Fig. 3, depending on the hop length, the meshed multipath is divided into three stages. Stage 1 covers the nodes from the source up to those $\lfloor H / 2\rfloor$ hops away, Stage 2 covers hops between $\lfloor H / 2\rfloor$ and $H-1$, and Stage 3 is the last hop. Successful packet arrival probability at the end of each stage, denoted by $P_{s}(i)$, where $i=1$ and 2, is first obtained as follows:

Stage 1: In this stage, a packet successfully reaches the next node if at least one of two downstream nodes is ready to receive, with probability $\left(1-p_{n}^{2}\right)$, and the channel is good during the packet transmission, with probability $\left(1-p_{l}\right)$. Since Stage 1 has $\lfloor H / 2\rfloor$ hops, $P_{s}(1)$ is given by

$P_{s}(1)=\left[\left(1-p_{l}\right)\left(1-p_{n}^{2}\right)\right]^{\lfloor H / 2\rfloor}$.

The probability with which a successful packet arrives at a node $N_{h, i+1}$ at the end of Stage 1 is binomially distributed:

$P_{h, i+1}=\frac{1}{2^{h}}\left(\begin{array}{c}h \\ i\end{array}\right)$

where $h=\lfloor H / 2\rfloor$ and $i=0,1, \ldots, h$.

Stage 2: $P_{S}(2)$ is obtained recursively as shown in Appendix A. Note that one needs to take into consideration up to three different cases depending on whether $H$ is odd or even, and if $H$ is odd, whether $\lfloor H / 2\rfloor$ is odd or even as illustrated in Fig. 3(a)-(c)). Also, the edge nodes beyond $\lceil H / 2\rceil$ hops (e.g., $N_{43}$ in Fig. 3(a)) have only one downstream node.

Finally, counting Stage 3 (i.e., the last hop), the end-to-end successful arrival probability of a packet, or normalized throughput is given by

$T_{\mathrm{SF}}^{(m)}=\left(1-p_{l}\right) \prod_{i=1}^{2} P_{s}(i)$

Note that, instead of the $H$-hop meshed multipath in Fig. 3, if Fig. 2(b) is considered (which illustrates a meshed multipath with the same number of nodes as in disjoint multipath shown in Fig. 2(a)), the throughput can be obtained in a straight forward way. Particularly, the first hop success probability is given by $P_{1}=\left(1-p_{n}^{r}\right)\left(1-p_{l}\right)$. For any $h$ from 2 to $H-1, P_{h}=P_{h-1}\left(1-p_{n}^{2}\right)\left(1-p_{l}\right)$ is obtained recursively. Finally, the normalized throughput is obtained as $T_{\mathrm{SF}}^{(m)}=P_{H-1}\left(1-p_{l}\right)$. This configuration will be considered in Section 4.2.2 for performance comparison between D-MPR and M-MPR.

Numerical and simulation results are provided in the next section.

\section{Performance results}

In this section, we first present the numerical results from throughput analysis and verify them via discrete event simulation. Subsequently, we will compare different MPR schemes in terms of resource usage (e.g., energy or bandwidth consumption). The intermediate nodes are assumed to fail intermittently (with probability $p_{n}$ ). If a node is found ready to receive before transmitting a packet (based on a priori local neighborhood information), it remains ready throughout the packet transmission period. However, channel noise can still corrupt a packet (with BER $p_{b}$ ). In studying the basic packet throughput performance, no attempt is made to correct packet error and all corrupted packets are discarded. However, FEC will be considered when comparing resource requirements of various schemes.

Unless otherwise specified, the parameter values considered in the simulation are the following: Number of nodes is 500, uniformly randomly distributed over a $500 \times 700 \mathrm{~m}$ location space; the range of circular coverage of each node is $40 \mathrm{~m}$; packet size is 50 Bytes (fixed); number of packets per session is $10^{6}$; link error probability $p_{l}$ is close to $10^{-3}$, calculated based on white Gaussian channel with BER $10^{-6}$, correspondingly $K=7$, $C=127$, and $E_{b} / N_{0}=17 \mathrm{~dB}$ (in Eq. (4)); node error probability $p_{n}$ varies and may be much higher than $p_{l}$ because unlike in MANET, while the sensor nodes are mostly stationary (and accordingly, $p_{l}$ is relatively smaller), they have a much more limited power and buffer space (and accordingly $p_{n}$ could be relatively higher).

Sufficient number of sessions are simulated to achieve throughput within a $95 \%$ confidence interval. Since in the simulation, end-to-end distance and meshed multipath formation vary widely for each session, instead of quantitative verification, 
we compare the analytically obtained performance trends with those from simulations.

\subsection{PR versus $S F$}

In studying relative performances of the PR and SF approaches, first, we consider normalized throughput. Then, we look into the resource usage, which is also of major interest from an energy efficiency view point, particularly in wireless sensor networks.

\subsubsection{Throughput performance}

Analytically obtained throughput performances of D-MPR and (its node-equivalent) M-MPR with PR and SF, respectively, for varying node failure probabilities, are shown in Fig. 4, which shows that PR has a higher normalized throughput than $\mathrm{SF}$ in D-MPR as well as in M-MPR. This is expected as sending a packet along multiple errorprone routes (rather than along one route) increases the chance of successful arrival of at least one copy of the packet.

Fig. 5 shows simulation-based throughput as a function of the node failure rate, with average endto-end distance of about 9 hops. Note that although the trends of results are similar as in Fig. 4, simulation gives a little poorer throughput performance because of the longer average hop

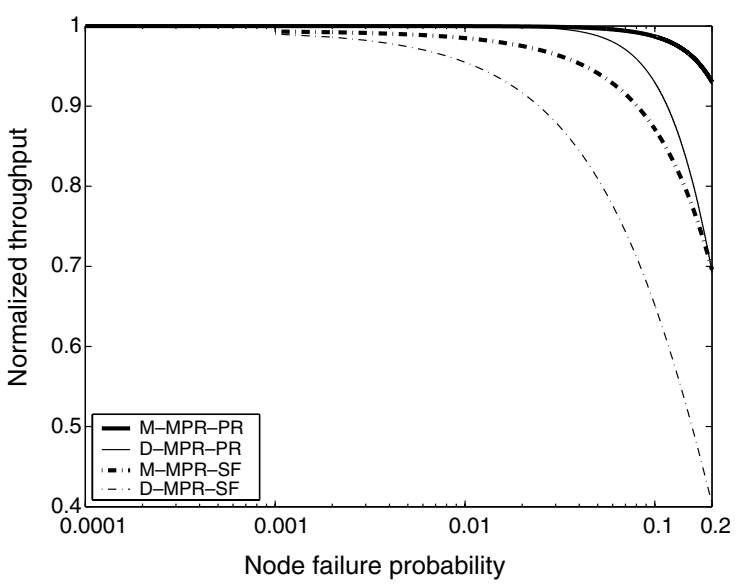

Fig. 4. Normalized throughput performances with PR and SF, respectively-from analysis. $p_{l}=10^{-3}, H=6$, and $r=3$.

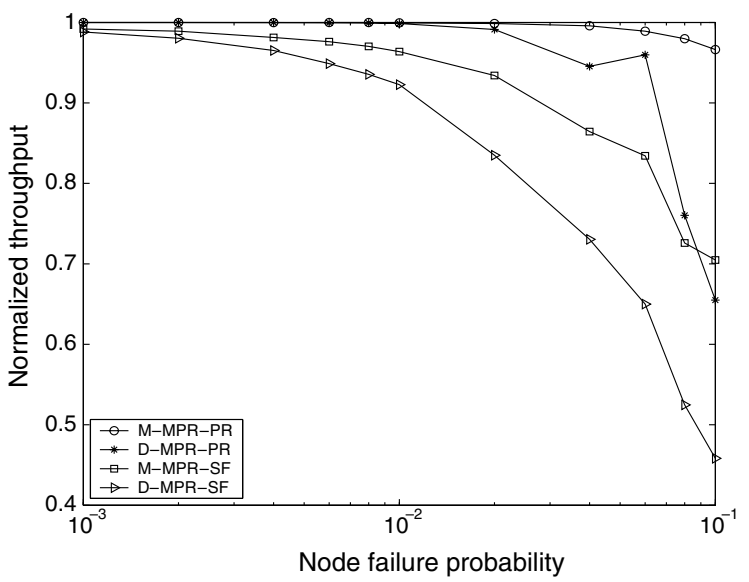

Fig. 5. Normalized throughput performance with PR and SF, respectively - from simulation. Average end-to-end distance is 9.06 hops.

length, irregular mesh, and unequal hop distance of multiple routes.

\subsubsection{Equivalent resource requirements}

To compare the above four approaches on the same baseline, we define equivalent resource usage as the number of transmit and receive operations carried to successfully route a message, as such a number is closely related to the energy consumption as well as channel bandwidth consumption. In the following, we use $E$ to denote the energy consumption.

We first determine the total number of packets to be sent for a given message using FEC coding. Assume that a message consists of $D$ data blocks. In $\mathrm{PR}$, let $T_{\mathrm{PR}}$ be the normalized throughput in PR (obtained in Eqs. (5) and (6)), and $C_{\mathrm{PR}}$ be the number of error correction blocks required to correctly retrieve the message (i.e., all $D$ data blocks). The corresponding notations in $\mathrm{SF}$ are, respectively, $T_{\mathrm{SF}}$ and $C_{\mathrm{SF}}$. Then, by [3],

$\left(D+C_{\mathrm{PR}}\right)\left(1-T_{\mathrm{PR}}\right) \leqslant C_{\mathrm{PR}}$,

$\left(D+C_{\mathrm{SF}}\right)\left(1-T_{\mathrm{SF}}\right) \leqslant C_{\mathrm{SF}}$,

that is, as long as the number of corrupted blocks is less than the number of error correction blocks, the message can be fully recovered at the receiver. 
Taking the limiting cases and simplifying them, the minimum number of error correction blocks required in the two cases are

$C_{\mathrm{PR}}=\left\lceil\frac{D\left(1-T_{\mathrm{PR}}\right)}{T_{\mathrm{PR}}}\right\rceil$,

$C_{\mathrm{SF}}=\left\lceil\frac{D\left(1-T_{\mathrm{SF}}\right)}{T_{\mathrm{SF}}}\right\rceil$.

To determine the number of transmit and receive operations needed for each packet, we make the following observations: (1) To reach more than one neighbor, a node requires only one transmit operation, which is the same as that for reaching a single neighbor. (2) Only if a node is an intended receiver (which is known at the MAC level), does it undergo one receive operation per packet transmission. (3) In PR, all nodes constituting the multipath route (disjoint or meshed) undergo transmit and receive operations. It is assumed that in M-MPR-PR, if an intermediate node receives more than one copy of a packet (with the same packet ID), it forwards only one. This, in a way, controls the data implosion at the destination [17] and also saves battery power.

Denote the number of transmit and receive operations for end-to-end packet delivery by $T X$ and $R X$, respectively. Referring to the example of disjoint multipath in Fig. 2(a), its node-equivalent meshed multipath (having 22 nodes) shown in Fig. 2(b), and its link-equivalent meshed multipath (having 24 links) shown in Fig. 3(a), we see that for each packet delivery using packet replication, while D-MPR-PR requires $21 T X$ and $24 R X$, its node-equivalent M-MPR-PR requires $21 T X$ and $40 R X$, and its link-equivalent M-MPR-PR requires $15 T X$ and $24 R X$. On the other hand, D-MPR-SF requires $6 T X$ and $6 R X$, so do its node-equivalent and link-equivalent M-MPR-SF.

Assume that the energy spent for a one hop packet transmission and its reception are nearly equal. ${ }^{2}$ Then, the equivalent energy spent per endto-end packet delivery is $T X+R X$. With these

\footnotetext{
${ }^{2}$ For unequal transmit and receive energies, $T X$ will be multiplied by a constant factor, determined by the ratio of transmit energy to receive energy.
}

observations, equivalent energy resource required to deliver the same message with PR and SF are obtained as

$$
\begin{aligned}
& E_{\mathrm{PR}}=\left(D+C_{\mathrm{PR}}\right)\left(T X_{\mathrm{PR}}+R X_{\mathrm{PR}}\right), \\
& E_{\mathrm{SF}}=\left(D+C_{\mathrm{SF}}\right)\left(T X_{\mathrm{SF}}+R X_{\mathrm{SF}}\right) .
\end{aligned}
$$

Table 1 shows the number of error correction blocks and the equivalent (energy) resource requirements for disjoint multipath (involving 15 nodes, with $H=6$ and $r=3$ ), ${ }^{3}$ as well as its nodeequivalent meshed multipath involving 14 nodes (shown in Fig. 3(a)), with PR and SF, respectively. For example, from the third row of the table, we see that for a given $p_{l}=10^{-3}, p_{n}=10^{-1}$, and $H=6$ hops, to successfully deliver a 1000 block long message, D-MPR-SF requires 535 error correction blocks $(C)$ and the associated equivalent energy usage is 18420 (units) (using Eq. (12)). In the identical scenario, D-MPR-PR requires only 76 error correction blocks, but 36584 units of equivalent energy usage, which is nearly double the required resource in D-MPR-SF. Correspondingly, M-MPR-PR requires 39546 units of energy resource, which is nearly 2.8 times that required in M-MPR-SF. It is apparent that PR wastes more network resources (in terms of battery power as well as channel bandwidth) compared to the SF, for achieving the same error performance limit, although SF needs more error correction blocks per message.

To verify the equivalent energy requirement $(E)$ via simulation, we obtain from the simulation trace file the disjoint multipath and meshed multipath for a specific source-destination pair (nodes 282 and 128) that are at least 6 hops away, as shown in Fig. 6.

For this specific case, the number of error correction blocks and the associated equivalent energy resource required with PR and SF in D-MPR and M-MPR, respectively, are shown in Table 2. Note

\footnotetext{
${ }^{3}$ We could have compared the disjoint multipath shown in Fig. 2(a) having $r=4$ disjoint routes with its node-equivalent meshed multipath shown in Fig. 2(b). Instead, we pick $r=3$ so as to be able to compare with the results from simulation later, where the disjoint multipath formed, shown in Fig. 6, has only $r=3$ disjoint paths.
} 
Table 1

Equivalent energy resource $(E)$ required with $\mathrm{PR}$ and SF, respectively-from analysis

\begin{tabular}{|c|c|c|c|c|c|c|c|c|}
\hline \multirow[t]{2}{*}{$p_{n}$} & \multicolumn{4}{|c|}{ D-MPR $(H=6, r=3)$} & \multicolumn{4}{|c|}{ M-MPR (Fig. 3(a)) } \\
\hline & $C_{\mathrm{PR}}^{(d)}$ & $E_{\mathrm{PR}}^{(d)}$ & $C_{\mathrm{SF}}^{(d)}$ & $E_{\mathrm{SF}}^{(d)}$ & $\overline{C_{\mathrm{PR}}^{(m)}}$ & $E_{\mathrm{PR}}^{(m)}$ & $C_{\mathrm{SF}}^{(m)}$ & $E_{\mathrm{SF}}^{(m)}$ \\
\hline $10^{-3}$ & 1 & 34034 & 11 & 12132 & 1 & 39039 & 7 & 12084 \\
\hline $10^{-2}$ & 1 & 34034 & 48 & 12576 & 1 & 39039 & 16 & 12192 \\
\hline 0.1 & 76 & 36584 & 535 & 18420 & 14 & 39546 & 147 & 13764 \\
\hline 0.2 & 443 & 49062 & 1476 & 29712 & 85 & 42315 & 433 & 17196 \\
\hline
\end{tabular}

$D=1000, H=6, p_{l}=10^{-3}$.

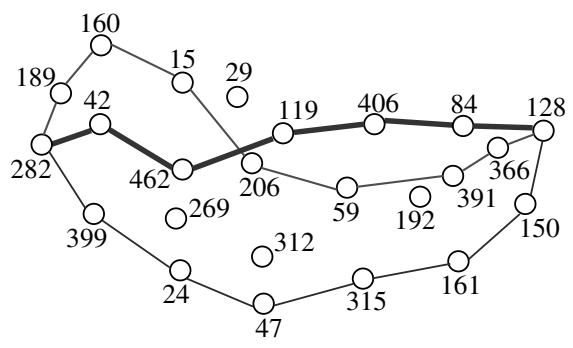

(a)

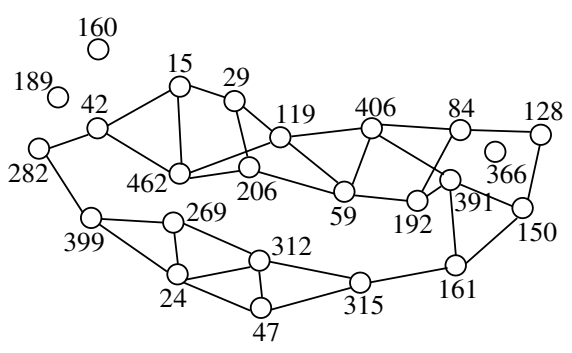

(b)

Fig. 6. Sketches of disjoint multipath and its node-equivalent meshed multipath, drawn from the network connectivity trace. Endto-end (shortest) distance 6 hops.

Table 2

Equivalent energy resource required with $\mathrm{PR}$ and SF, respectively-from simulation

\begin{tabular}{|c|c|c|c|c|c|c|c|c|}
\hline \multirow[t]{2}{*}{$p_{n}$} & \multicolumn{4}{|c|}{ D-MPR (Fig. 6(a)) } & \multicolumn{4}{|c|}{ M-MPR (Fig. 6(b)) } \\
\hline & $\overline{C_{\mathrm{PR}}^{(d)}}$ & $E_{\mathrm{PR}}^{(d)}$ & $C_{\mathrm{SF}}^{(d)}$ & $E_{\mathrm{SF}}^{(d)}$ & $\overline{C_{\mathrm{PR}}^{(m)}}$ & $E_{\mathrm{PR}}^{(m)}$ & $C_{\mathrm{SF}}^{(m)}$ & $E_{\mathrm{SF}}^{(m)}$ \\
\hline $10^{-3}$ & 0 & 40000 & 8 & 14112 & 1 & 54054 & 5 & 16080 \\
\hline $10^{-2}$ & 1 & 40040 & 55 & 14770 & 1 & 54054 & 21 & 16336 \\
\hline 0.1 & 186 & 47440 & 717 & 24038 & 35 & 55890 & 261 & 20176 \\
\hline 0.2 & 1110 & 84400 & 2280 & 45920 & 188 & 64152 & 773 & 28368 \\
\hline
\end{tabular}

End-to-end (shortest) distance 6 hops. $D=1000, p_{l}=10^{-3}$.

that since "equal length routes" and "ideal mesh" could not be ensured in the simulation (due to random location of nodes), to route a message to the destination, the number of transmit-receive operations obtained from simulation is higher than the corresponding number obtained analytically, resulting in a higher $E$. Nevertheless, as shown in Fig. 7, in terms of the savings in the equivalent energy resource usage due to SF (over PR) in DMPR and M-MPR, respectively, calculated from the data in Tables 1 and 2, the results obtained from analysis follow closely those from simulations.
Given that for a successful message transmission PR has much higher energy resource overhead compared to the SF (even though PR has a higher packet throughput), in the subsequent discussions, we will concentrate only on the SF approach.

\section{2. $M-M P R-S F$ versus $D-M P R-S F$}

From the analytical results (columns 5 and 9 in Table 1), we can see that when the node failure rate $p_{n}$ is low, D-MPR-SF consumes only slightly 


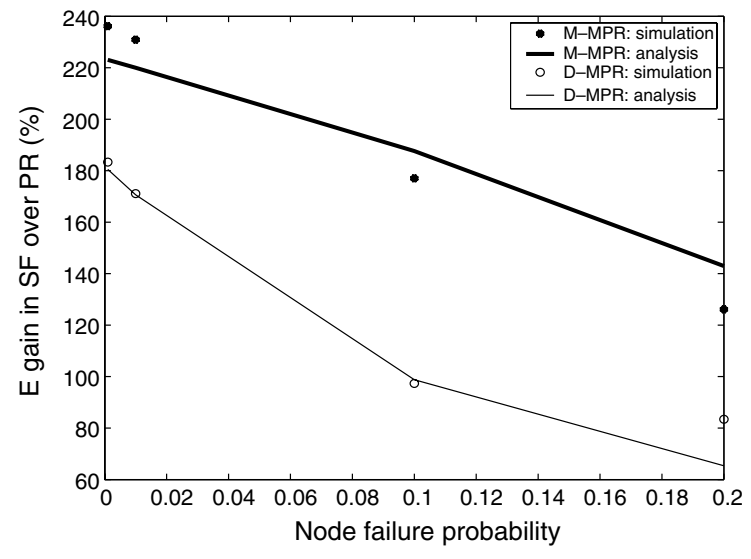

Fig. 7. Equivalent energy resource $(E)$ gain with $\mathrm{SF}$ over $\mathrm{PR}$, in D-MPR and M-MPR, respectively. End-to-end (shortest) distance 6 hops.

more energy than its node-equivalent M-MPR-SF to deliver the same message. However, the difference becomes quite significant when $p_{n}$ becomes large. This is because although D-MPR-SF and M-MPR-SF undergo the same number of $T X$ and $R X$ operations along a given path (of equal length), D-MPR-SF has a poorer packet throughput (or packet loss probability), and accordingly, it would require more error correction blocks and hence more energy for successfully delivering a message than with M-MPR-SF.

A further look at the simulation results (columns 5 and 9 in Table 2) reveals that when $p_{n}$ is low, M-MPR-SF consumes a little more energy for successfully delivering a message than D-MPR-SF. This is because in simulation, a packet may undergo a longer path in M-MPR-SF than in DMPR-SF (see Fig. 6 where M-MPR-SF may use a 7- or 8-hops path while D-MPR-SF will only use a 6-hop path), and accordingly, requiring a larger number of TX and RX operations. However, as in the analysis, when $p_{n}$ increases, the energy requirement in D-MPR-SF increases at a much faster rate compared to M-MPR-SF due to the fact that the former requires a much larger number of error correction blocks than the latter. Eventually, the energy requirement of D-DPR-SF surpasses that of M-MPR-SF. Note that, this also explains why in the case of packet-replication
(PR), D-MPR-PR also consumes more energy than M-MPR-PR when $p_{n}$ is large enough. ${ }^{4}$

Additional advantages of M-MPR-SF are shown in the following subsections.

\subsubsection{Throughput gain}

To compare the throughput of M-MPR-SF with its node-equivalent D-MPR-SF, we determine the number of disjoint routes, $r$, in D-MPR, so that the number of nodes in M-MPR is approximately equal to the number of nodes in D-MPR. Considering the routes in Fig. 2, the analytic throughput gain in M-MPR-SF over its node-equivalent D-MPR-SF is shown in Fig. 8, where it is apparent that the improvement of MMPR-SF over D-MPR-SF increases as the route gets longer. As a reason for the poorer performance of D-MPR-SF, we note that once a route is decided at the source end, no further alternate routing option is available. Hence, any failure at the intermediate stage implies packet loss. On the other hand, in M-MPR-SF, routing flexibility is available throughout the route.

Simulation-based results on the normalized throughput of D-MPR-SF and its node-equivalent M-MPR-SF as a function of end-to-end distance, averaged over a number of simulation runs, is shown in Fig. 9. The average source-destination hop length is varied by changing the aspect ratio of the location space. For the same aspect ratio of the location space, the difference in average hop length in disjoint and meshed MPR scenarios is caused by the randomness of node locations. Hence, throughput gain could not be computed directly. However, the slopes of normalized throughput (the straight lines, obtained by interpolation) in the two cases indicate a higher gain in M-MPR-SF for a longer route. The results on the improvement of M-MPR-SF over its link-equivalent D-MPR$\mathrm{SF}$ are similar and hence omitted because of space limitations.

\footnotetext{
${ }^{4}$ The reason that D-MPR-PR consumes less energy than MMPR-PR when $p_{n}$ is small in both analysis and simulation is that in the former, a packet goes through a fewer TX and RX operations because a disjoint multipath contains a fewer links than a node-equivalent meshed multipath (see, e.g., Figs. 2(a) and 3(a)).
} 


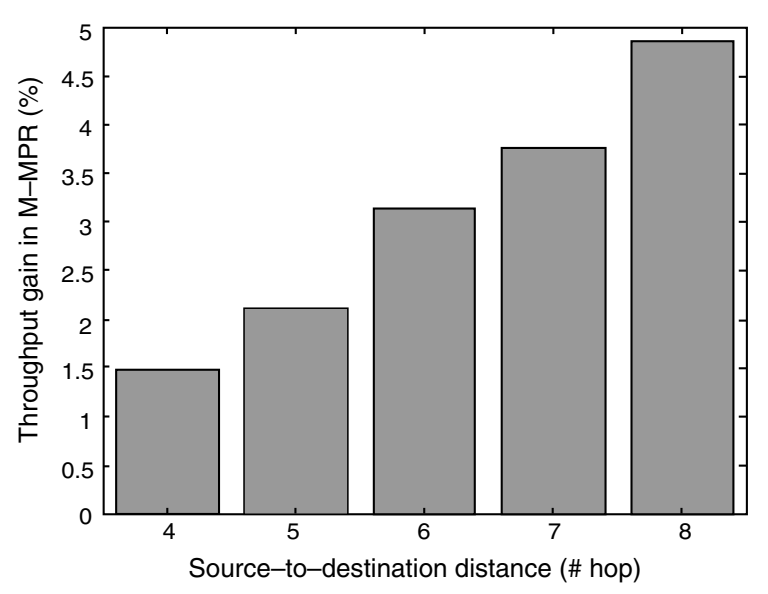

Fig. 8. Percentage throughput gain in M-MPR-SF over its node-equivalent D-MPR-SF scheme-from analysis. $p_{l}=10^{-3}$, $p_{n}=10^{-2}$.

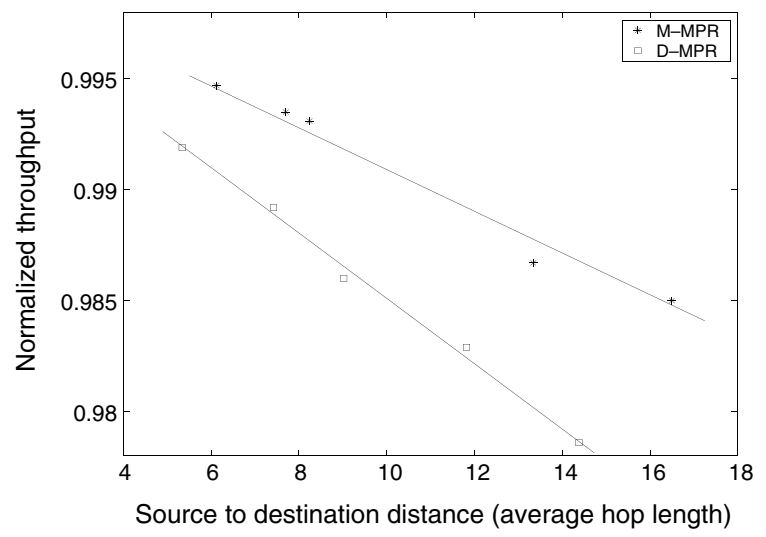

Fig. 9. Throughput variations of D-MPR-SF and its nodeequivalent M-MPR-SF with end-to-end distance-from simulation. $p_{n}=10^{-3}$.

\subsubsection{Receiver complexity}

To compare the receiver complexity, without loss of generality, we assume Direct Sequence Spread Spectrum (DS-SS) based medium access, where each node has its unique (orthogonal) code for transmission. We do not consider spatial separation dependent code reuse. Therefore, the number of orthogonal codes required is equal to the number of transmitting nodes $(N)$ along the route, and the number of correlators required in a receiver is equal to the number of incoming links $(L)$. The total number of correlators required in a multipath route determines the receiver complexity of the routing scheme.

Considering M-MPR-SF and its node-equivalent as well as link-equivalent D-MPR-SF, Fig. 10 shows the analytically obtained normalized throughput of 6-hops routes shown in Figs. 2(a), (b) and 3(a). We note that in the node-equivalent case (e.g., shown in Fig. 2(a) and (b), where $\left.N^{(d)}=N^{(m)}=22\right)$, although M-MPR-SF has a much higher throughput, it has a higher receiver complexity as well $\left(L^{(m)}=40\right.$ versus $\left.L^{(d)}=24\right)$. However, in the link-equivalent case (Figs. 2(a) and 3(a) where $L^{(d)}=L^{(m)}=24$ ), M-MPR-SF still achieves a better throughput than D-MPR-SF, even though the former involves a fewer nodes $\left(N^{(m)}=16\right.$ versus $\left.N^{(d)}=22\right)$ and thus a lower receiver complexity.

Fig. 11 plots simulation results on normalized throughput, where the end-to-end distance is about 9 hops, averaged over multiple sessions. We observe that the trend is similar to that from the analysis as shown in Fig. 10. Note that due to random placement of nodes, one can no longer ensure idealized mesh and equal length multiple routes (e.g., in Figs. 2 and 3), which, coupled with longer average hop length, leads to poorer performance from simulation than that from analysis.

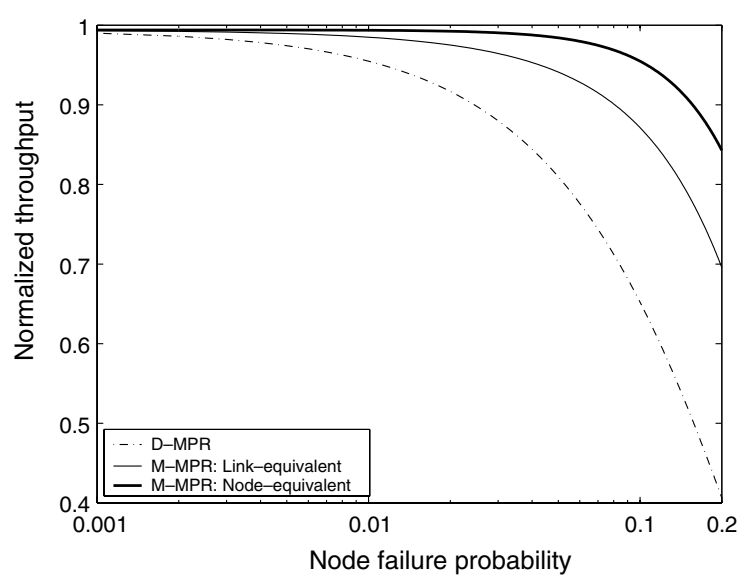

Fig. 10. Normalized throughput performance of D-MPR-SF and its equivalent M-MPR-SF schemes-from analysis. $p_{l}=10^{-3}, H=6$. 


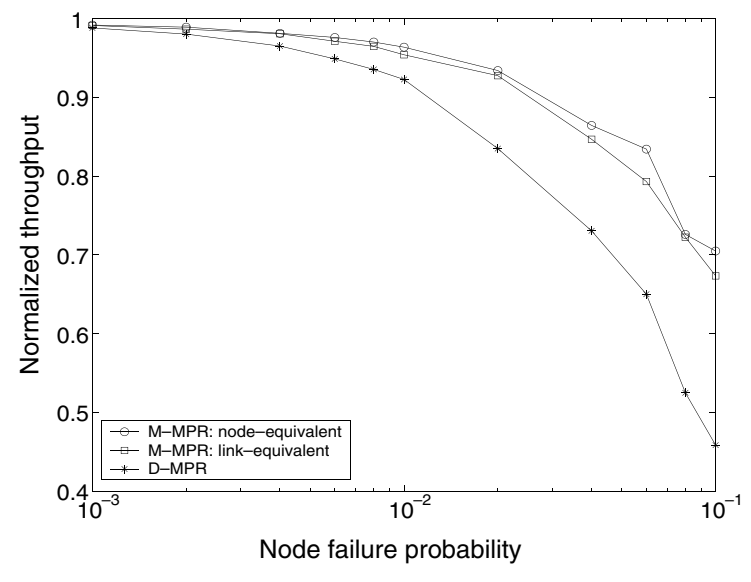

Fig. 11. Normalized throughput performance of D-MPR-SF and its equivalent M-MPR-SF schemes, obtained from simulations. Average number of hops is 9.06 .

\section{Related work}

There have been numerous proposals on multipath routing in interconnection networks for either high-speed operation or failsafe communication. We briefly survey the related work and highlight our contributions in this paper.

\subsection{Route discovery}

In conventional single route or multiple route searching strategies, one end node (e.g., the source) sends route query (or discovery) packets to the other end (e.g., the destination) via flooding $[21,25,27]$, or scoped flooding $[8,16]$ with a preset time-to-live (or hop count) value. In DSR-like [14] route discovery approaches, each discovery packet records the partial route it has followed so far $[8,27]$. For a source-to-destination route, an intermediate node entertains only one discovery packet and forwards it to its downstream neighbors, thus forming a source tree towards the destination. The destination, upon receiving the discovery packets, replies to either one or multiple of them with reservation confirmation. Such an approach creates either disjoint multiple routes or a primary route. If only a single (i.e., primary) route is established at the first route search phase, disjoint secondary routes can be formed sequentially [12] (by removing already established routes). To set up braided multipath around the primary route (i.e., having non-disjoint secondary routes), for each node along the primary route, an alternate route is discovered sequentially [12]. In either case, such a multipath searching approach would require high control overhead and associated delay. Alternatively, in distributed route searching (e.g., AODV [25], AOMDV [21]), instead of the packet carrying the entire route information, each involved node maintains its upstream and downstream nodes for forward and reverse path. In AODV [25], a single path is searched via tree-based query flooding, where at most one discovery packet (and the corresponding route) is accepted by a node. In AOMDV [21], the intermediate nodes are allowed to receive more than one discovery packet, thereby forming linkdisjoint multiple routes. But the route searching is still done via flooding (which results in high network-wide control overhead and battery power consumption).

Our meshed multipath searching approach is similar to AOMDV [21]. However, in view of limited battery power and available location information of nodes in sensor networks, our approach has the following distinct features: (a) For route discovery from each source we restrict to no more than two best neighbors for discovery packet forwarding. (b) Because of many-sources-to-onedestination route discovery, routing table and discovery packet lengths are reduced. (c) To reduce power consumption, a node forwards only one of possibly many discovery packets, received from its peripheral sources, to the destination. (d) Destination-to-many-sources route reply is sent via multicasting.

\subsection{Data packet routing}

The authors in [17] presented different approaches for improving on a simple flooding technique for sensor networks by introducing node-to-node co-ordination, thereby reducing chances of overlapped data collection and data implosion. In [18], multicasting along mesh-based routes to a group of nodes in multihop wireless networks has been proposed. Packet replication along a meshed multipath is similar to the dis- 
tributed parallel processing in bus interconnection networks [6], where the data to be operated on is copied to all the operators (networks nodes); thus faster computation speed is achieved at the cost of communication bandwidth and nodal memory consumption. In mobile ad hoc networks and sensor networks, to ensure delay and/or loss guarantee, multiple disjoint [8] or partially disjoint $[12,23]$ routes are set up, and data is transmitted along primary routes while the unused secondary routes are maintained via periodic control signaling. To deal with network error, either end-to-end [8] or adjacent node [12,29] acknowledgment (or negative acknowledgment) based rerouting is done. Traffic splitting along disjoint multiple routes [19] (called disjoint multipath routing, or DMPR) is aimed at network load balancing. For a given channel error probability, [28] studied the optimum number of disjoint multiple routes to ensure successful data delivery. Directed diffusion approach [13] set up a single-path route from sink to the source based on the interest gradient of data. Credit-based mesh forwarding [30] introduced flexibility of a single-path route selection to address dynamic network conditions. Only one of multiple routes, called the primary route, is used for data transmission.

The distinct features of our meshed multipath routing (M-MPR) over the existing multipath approaches are the following: (a) As opposed to PR approach [17], a packet is forwarded along only one selected next hop node. (b) Instead of splitting traffic along disjoint multipaths [19,28], meshed multipath introduces more flexibility in on-the-fly routing decisions. (c) Instead of sending traffic along a preferential (primary) route among a number of disjoint or partially disjoint multiple alternatives [8,12,13,21,23], M-MPR distributes traffic more evenly in the mesh, thereby achieving better load balancing and requiring less signaling overhead to deal with link or node failure and for multiple route maintenance. (d) Unlike in $[5,8,12,15,29]$, the absence of acknowledgmentbased retransmission and rerouting is aimed at a simplified flow control mechanism, and reduced buffer requirements, additional transmit-to-receive mode changeover delay, and receive power consumption at the field sensors.

\section{Conclusion}

We have presented a meshed multipath routing scheme with selective packet forwarding for wireless sensor networks. The routing decision is taken dynamically, hop-by-hop, based on the conditions of downstream forwarding nodes. End-to-end FEC coding is used to avoid acknowledgmentbased retransmission. Our aim has been to ensure successful data communication with minimal buffering and flow control overhead, and efficient use of network resources such as bandwidth and battery power. The proposed routing strategy is a more natural choice in multihop wireless sensor networks, which have high nodal density, and where each node has only partial network (local) information, limited power, and limited functionality.

We have outlined the meshed multipath discovery and routing strategies. Performance of the proposed protocol has been evaluated and compared with the existing competitive approaches analytically as well as via simulations. Our evaluation has shown that although packet replication (or limited flooding) over multiple paths has a higher packet level throughput compared to selective forwarding, the latter requires much less network resources for successfully delivering a message. We have shown significant improvement in throughput performance with the proposed meshed multipath routing scheme over its nodeand link-equivalent disjoint multipath, without consuming additional network resources. Overall, the proposed meshed multipath routing with selective forwarding achieves a superior performance.

\section{Appendix A. Calculation of $\boldsymbol{P}_{s}(2)$ in M-MPR-SF} (9).

$P_{\lfloor H / 2\rfloor, i+1}, 0 \leqslant i \leqslant\lfloor H / 2\rfloor$, is obtained from Eq.

BEGIN

IF $H$ odd, FOR $j=1$ through $\lfloor H / 2\rfloor$, with increment 2 ,

$$
P_{\lceil H / 2\rceil, j} \leftarrow \frac{P_{[H / 2], j}+P_{[H / 2], j+1}}{2}\left(1-p_{n}^{2}\right)\left(1-p_{l}\right)
$$




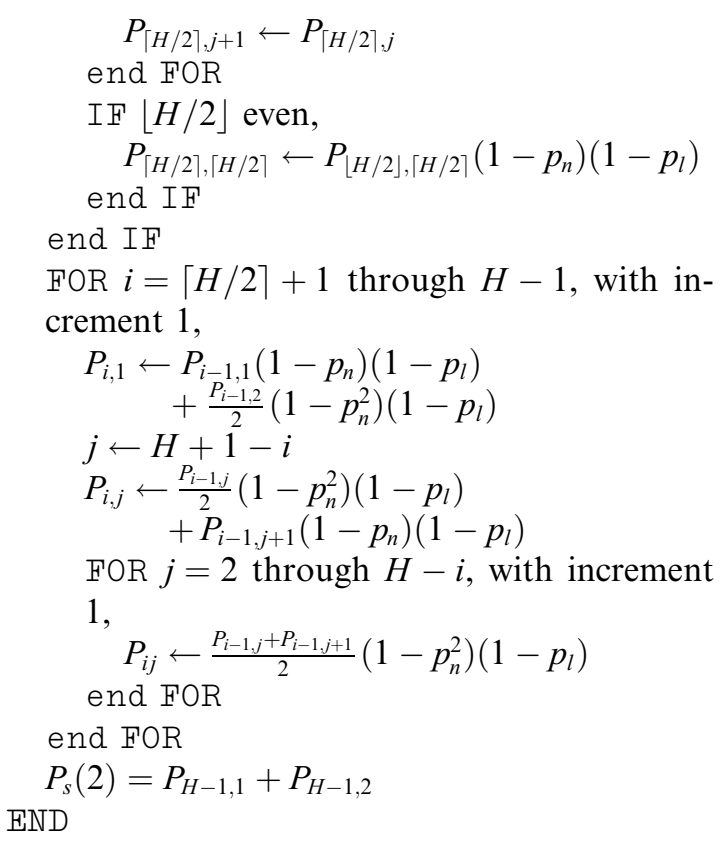

\section{References}

[1] I.F. Akyildiz, W. Su, Y. Sankarasubramaniam, E. Cayirci, Wireless sensor networks: a survey, Computer Networks 38 (4) (2002) 393-422.

[2] G. Asada, M. Dong, T.S. Lin, F. Newberg, G. Pottie, W.J. Kaiser, H.O. Marcy, Wireless integrated network sensors: low power systems on a chip, in: Proc. European Solid State Circuits Conf., 1998.

[3] E. Ayanoglu, I. Chih-Lin, R.D. Gitlin, J.E. Mazo, Diversity coding for transparent self-healing and fault-tolerant communication networks, IEEE Transactions on Communications 41 (11) (1993) 1677-1686.

[4] A. Banerjea, Simulation study of the capacity effects of dispersity routing for fault tolerant real-time channels, ACM Computer Communication Review 26 (1996) 194 205.

[5] R.V. Boppana, S. Chalasani, Fault-tolerant wormhole routing algorithms for mesh networks, IEEE Transactions on Computers 44 (7) (1995) 848-864.

[6] N. Carriero, D. Gelernter, The S/Net's Linda Kernel, ACM Transactions on Computer Systems 4 (2) (1986) 110 129.

[7] A. Chandrakasan, R. Amritharajah, S. Cho, J. Goodman, G. Konduri, J. Kulik, W. Rabiner, A. Wang, Design considerations for distributed microsensor systems, in: IEEE Custom Integrated Circuits Conf., May 1999, pp. 279-286.

[8] S. Chen, K. Nahrstedt, Distributed quality-of-service routing in ad hoc networks, IEEE Journal on Selected Areas in Communications 17 (8) (1999) 1488-1505.
[9] L.P. Clare, G.J. Pottie, J.R. Agre, Self-organizing distributed sensor networks, in: SPIE Conf. Unattended Ground Sensor Technologies and Applications, April 1999, pp. 229-237.

[10] S. De, S.K. Das, H. Wu, C. Qiao, Trigger based distributed QoS routing in mobile ad hoc networks, ACM SIGMOBILE Mobile Computing and Communications Review 6 (3) (2002) 22-35.

[11] P. Enge, P. Misra (Eds.), Special Issue on GPS: The Global Positioning System, Proc. IEEE 87 (1) (1999) 3-172.

[12] D. Ganesan, R. Govindan, S. Shenker, D. Estrin, Highlyresilient, energy-efficient multipath routing in wireless sensor networks, ACM SIGMOBILE Mobile Computing and Communications Review 5 (4) (2001) 11-25.

[13] C. Intanagonwiwat, R. Govindan, D. Estrin, Directed diffusion: a scalable and robust communication paradigm for sensor networks, in: Proc. ACM MobiCom, 2000.

[14] D.B. Johnson, D.A. Maltz, Y.-C. Hu, J.G. Jetcheva, The dynamic source routing protocol for mobile ad hoc networks (DSR), IETF Internet draft, draft-ietf-manetdsr-07.txt, February 2002.

[15] P. Kermani, L. Kleinrock, Virtual cut-through: a new communication switching technique, Computer Networks 3 (11) (1979) 267-286.

[16] Y.-B. Ko, N.H. Vaidya, Location-aided routing (LAR) in mobile ad hoc networks, in: Proc. ACM MobiCom, 1998.

[17] J. Kulik, W.R. Heinzelman, H. Balakrishnan, Negotiationbased protocols for disseminating information in wireless sensor networks, in: Proc. ACM MobiCom, 1999.

[18] S.-J. Lee, M. Gerla, C.-C. Chiang, On-demand multicast routing protocol, in: Proc. IEEE WCNC, September 1999, pp. 1298-1302.

[19] S.-J. Lee, M. Gerla, Split multipath routing with maximally disjoint paths in ad hoc networks, in: Proc. Int. Conf. Commun. (ICC), 2001.

[20] S. Madden, M.J. Franklin, J.M. Hellerstein, W. Hong, The design of an acquisitional query processor for sensor networks, in: Proc. ACM SIGMOD, June 2003.

[21] M.K. Marina, S.R. Das, On-demand multipath distance vector routing in ad hoc networks, in: Proc. Int. Conf. Network Protocols (ICNP), November 2001.

[22] N.F. Maxemchuk, Dispersity routing in high-speed networks, Computer Networks and ISDN Systems 25 (1993) 645-661.

[23] A. Nasipuri, S.R. Das, On-demand multipath routing for mobile ad hoc Networks, in: Proc. Int. Conf. Comput. Commun. Networks (ICCCN), October 1999, pp. 64-70.

[24] A. Nasipuri, K. Li, A directionality based location discovery scheme for wireless sensor networks, in: Proc. ACM Workshop on Wireless Sensor Networks and Applications (WSNA), September 2002, pp. 105-111.

[25] C.E. Perkins, E.M. Royer, Ad hoc on-demand distance vector routing, in: Proc. 2nd IEEE Workshop on Mobile Computing Systems and Applications, February 1999, pp. 90-100.

[26] T. Rappaport, Wireless Communications: Principles and Practice, Prentice Hall, Englewood Cliffs, NJ, 1996. 
[27] W. Su, M. Gerla, IPv6 flow handoff in ad hoc wireless networks using mobility prediction, in: Proc. IEEE GLOBECOM, 1999.

[28] A. Tsirigos, Z.J. Hass, Multipath routing in the presence of frequent topological changes, IEEE Communications Magazine 39 (11) (2001) 132-138.

[29] C.-Y. Wan, A.T. Campbell, L. Krishnamurthy, PSFQ: a reliable transport protocol for wireless sensor networks, in: Proc. ACM Workshop on Wireless Sensor Networks and Applications (WSNA), September 2002, pp. 1-11.

[30] F. Ye, S. Lu, L. Zhang, GRAdient broadcast: a robust, long-lived large sensor network, in: UCLA CS Technical Report, September 2001.

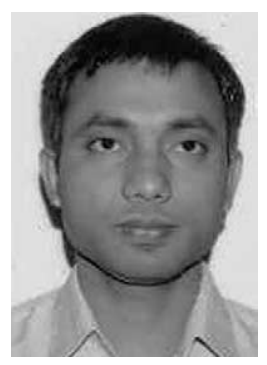

Swades De received his B.Tech degree in Radiophysics and Electronics from University of Calcutta in 1993 and his M.Tech degree in Optoelectronics and Optical Communication from Indian Institute of Technology Delhi in 1998. During 1993-1997 he was a hardware development engineer and in the first half of 1999 he was a software engineer in different telecommunication companies in India. He is a Ph.D candidate in the Electrical Engineering Department at State University of New York at Buffalo. His current research interests include performance study, QoS routing and resource optimization in mobile ad hoc networks and wireless sensor networks, integrated wireless technologies, multipath routing in high-speed networks, and communications and systems issues in optical networks.

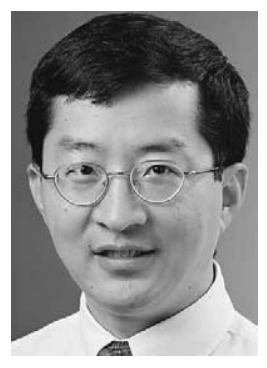

Chunming Qiao is an Associate Professor at the University at Buffalo (SUNY), where he directs the Lab for Advanced Network Design, Analysis, and Research (LANDER), that conducts cutting-edge research work on optical networks, wireless networks, and the Internet. He has published more than one hundred forty papers in leading technical journals and conference proceedings, and is recognized for his pioneering research on Optical Internet, in particular, the optical burst switching (OBS) paradigm. His work on integrated cellular and ad hoc networking systems (iCAR) is also internationally acclaimed and has been featured in Businessweek and Wireless Europe.

$\mathrm{He}$ is on the editorial board of several journals and magazines including IEEE Communications and IEEE/ACM Transactions on Networking (ToN), and has guest-edited three IEEE JSAC issues. He has chaired and co-chaired many international conferences and workshops including the HighSpeed Networking Workshop (formerly GBN) at Infocom'01 and Infocom'02, Opticomm'02, and the symposium on Optical Networks at ICC'03.

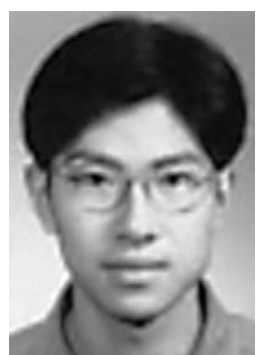

Hongyi Wu is currently a tenure-track Assistant Professor at The Center for Advanced Computer Studies (CACS), University of Louisiana (UL) at Lafayette. He received his Ph.D degree in Computer Science from State University of New York (SUNY) at Buffalo in 2002. He received his M.S. degree from SUNY at Buffalo in 2000 and B.S. degree from Zhejiang University in 1996, respectively. He worked in Nokia Research Center in the summers of 2001 and 2000. His research interests include wireless mobile ad hoc networks, sensor networks, the next generation cellular systems, and the integrated heterogeneous wireless systems. He has chaired the Integrated Heterogeneous Wireless Networks symposium in VTC 2003, and served as a technical program committee member of several conferences and the guest editor of ACM MONET special issue on Integration of Heterogeneous Wireless Technologies. He has published about two dozen technical papers in leading journals and conference proceedings, as well as a book chapter. 